\title{
99m Tc-anti-TNF- $\alpha$ antibody for the imaging of disease activity in pulmonary sarcoidosis
}

\author{
Roeland Vis ${ }^{1}$, Gaurav Malviya ${ }^{2,3}$, Alberto Signore ${ }^{3,4}$, Jan C. Grutters ${ }^{5,6}$, \\ Bob Meek ${ }^{7}$, Ewoudt M.W. van de Garde ${ }^{1}$ and Ruth G.M. Keijsers ${ }^{8}$
}

Affiliations: ${ }^{1}$ Dept of Clinical Pharmacy, St Antonius Hospital, Nieuwegein, The Netherlands. ${ }^{2}$ Nuclear Imaging, Cancer Research UK Beatson Institute, Glasgow, UK. ${ }^{3}$ Dept of Nuclear Medicine and Molecular Imaging, University Medical Centre Groningen, University of Groningen, Groningen, The Netherlands. ${ }^{4}$ Nuclear Medicine Unit, Dept of Medical-Surgical Sciences and of Translational Medicine, Faculty of Medicine and Psychology, "Sapienza" University of Rome, Rome, Italy. ${ }^{5}$ Centre of Interstitial Lung Diseases, Dept of Pulmonology, St Antonius Hospital, Nieuwegein, The Netherlands. ${ }^{6} \mathrm{Heart}$ and Lung Division, University Medical Centre Utrecht, Utrecht, The Netherlands. ${ }^{7}$ Dept of Medical Microbiology and Immunology, St Antonius Hospital, Nieuwegein, The Netherlands. ${ }^{8}$ Dept of Nuclear Medicine, St Antonius Hospital, Nieuwegein, The Netherlands.

Correspondence: Roeland Vis, Dept of Clinical Pharmacy, St Antonius Hospital, Koekoekslaan 1, 3435 CM Nieuwegein, the Netherlands. E-mail: r.visdantoniusziekenhuis.nl

ABSTRACT Infliximab, a monoclonal antibody directed against tumour necrosis factor (TNF)- $\alpha$, is used in the treatment of refractory sarcoidosis. However, the clinical response is variable and a tool to select responders beforehand is highly desirable. In this study we evaluated scintigraphy with technetium- $99 \mathrm{~m}$ $\left({ }^{99 \mathrm{~m}} \mathrm{Tc}\right)$-labelled infliximab for the imaging of disease activity in patients with pulmonary sarcoidosis.

10 patients were studied using single photon emission computed tomography/computed tomography (CT) $6 \mathrm{~h}$ and $20 \mathrm{~h}$ after intravenous administration of $370 \mathrm{MBq}$ of ${ }^{99 \mathrm{~m}} \mathrm{Tc}$-infliximab. Correlation analysis was performed between tissue accumulation of ${ }^{99 \mathrm{~m}} \mathrm{Tc}$-infliximab and laboratory parameters (including soluble interleukin-2 receptor and angiotensin-converting enzyme), lung function parameters (including forced expiratory volume in $1 \mathrm{~s}$ and the diffusing capacity of the lung for carbon monoxide) and ${ }^{18} \mathrm{~F}$ fluorodeoxyglucose (FDG) positron emission tomography (PET)/CT.

Analysis showed selective and variable accumulation of ${ }^{99 \mathrm{~m}} \mathrm{Tc}$-infliximab in the target tissue. Accumulation correlated positively with all four laboratory parameters and negatively with all four lung function parameters, yielding better correlations than serum TNF- $\alpha$ levels or ${ }^{18}$ F-FDG PET/CT.

${ }^{99 \mathrm{~m}} \mathrm{Tc}$-infliximab accumulation reflects the in situ TNF- $\alpha$ expression in an individual patient and therefore provides valuable information on the presence of the biological target for anti-TNF- $\alpha$ therapy.

@ERSpublications

${ }^{99} \mathrm{~m}$ Tc-TNF- $\alpha$ antibody accumulation reflects presence of the biological target for anti-TNF therapy in sarcoidosis http://ow.ly/V92cz 


\section{Introduction}

Sarcoidosis is a systemic, granulomatous disease of unknown aetiology. It frequently presents with bilateral hilar lymphadenopathy and pulmonary infiltration, but several other organs may also be involved [1]. Although the majority of patients will exhibit a spontaneous remission, severe organ involvement necessitates the use of immunosuppressive therapy with corticosteroids as first-choice drugs [2]. In cases of progressive deterioration or intolerance for steroids, second-line agents can be used, such as azathioprine and methotrexate [3].

Tumour necrosis factor (TNF)- $\alpha$ is a critical proinflammatory cytokine in the formation of the sarcoid granuloma and is primarily released from alveolar macrophages $[4,5]$. Increased but highly variable TNF- $\alpha$ levels are observed in the bronchoalveolar lavage (BAL) fluid and lymph nodes of sarcoidosis patients [5-7].

Infliximab, a chimeric monoclonal antibody directed against TNF- $\alpha$, has shown to be an effective therapy in several TNF- $\alpha$ mediated conditions, such as rheumatoid arthritis, inflammatory bowel disease (IBD) and ankylosing spondylitis [8-10]. In addition, several studies indicate that infliximab might be useful in the treatment of severe pulmonary and extrapulmonary sarcoidosis [11-14]. However, the clinical response is variable, with reported response rates between $38 \%$ and $88 \%$, depending on definitions, treatment duration and organ involvement $[13,15,16]$. Clinical characteristics identifying subsets of patients with better responses include disease severity, C-reactive protein, serum TNF- $\alpha$ and TNF- $\alpha$ G-308A polymorphism, but to date none of these parameters enables the prediction and subsequent selection of responders prior to the initiation of therapy [11, 17-19]. Considering the substantial costs, potential side-effects and risk of disease progression during infliximab nonresponse, a tool to select responders beforehand is highly desirable.

Infliximab has been labelled with technetium-99m $\left({ }^{99 \mathrm{~m}} \mathrm{Tc}\right)$ and used for in vivo imaging in several TNF- $\alpha$ mediated diseases such as rheumatoid arthritis, spondyloarthropathy, IBD and sarcoidosis [20-22]. In rheumatoid arthritis and spondylarthropathy patients the accumulation of ${ }^{99} \mathrm{~m}$ Tc-infliximab prior to intra-articular infliximab therapy allowed the identification of responders, partial responders and nonresponders [20]. In sarcoidosis, low pulmonary accumulation of ${ }^{99} \mathrm{~m}$ Tc-infliximab has been reported, with a moderate correlation with the CD4/CD8 ratio in peripheral blood lymphocytes [22]. Nevertheless, the scintigraphic quantification of TNF- $\alpha$ in sarcoidosis patients might provide an additional measure to evaluate disease activity and potentially enables the prediction of anti-TNF- $\alpha$ therapy response.

In the present study, we evaluated the role of ${ }^{99 \mathrm{~m}} \mathrm{Tc}$-infliximab scintigraphy for the imaging of disease activity in patients with pulmonary sarcoidosis.

\section{Material and methods}

\section{Study subjects}

10 newly diagnosed patients with pulmonary sarcoidosis were recruited at the St Antonius Hospital (Nieuwegein, the Netherlands) between November 2012 and February 2014. Patients were diagnosed with sarcoidosis in accordance with the guidelines of the World Association of Sarcoidosis and Other Granulomatous Disorders [1]. In cases of an indication for systemic treatment, this was initiated after study participation.

Laboratory analysis for all patients included soluble interleukin-2 receptor (sIL-2R) and angiotensin-converting enzyme (ACE), including Z-scores of ACE insertion-/deletion-corrected ACE activity [23].

Chest radiography was performed from an anterior and lateral view and scored in accordance with the Scadding criteria. ${ }^{18}$ F-Fluorodeoxyglucose (FDG) positron emission tomography (PET)/computed tomography (CT) was performed for routine work-up and in accordance with the European Association of Nuclear Medicine guidelines. Pulmonary function tests were performed, including the vital capacity (VC) and diffusing capacity of the lung for carbon monoxide (DLCO). All diagnoses were confirmed by either histological proof in biopsy or a confirmative CD4/CD8 ratio (>3.5) in the BAL.

The study was performed in accordance with the Declaration of Helsinki and its amendments. The protocol was approved by the regional medical ethics committee (EudraCT number 2011-003630-13), and written informed consent for participation was obtained from all patients.

\section{Scintigraphy}

Infliximab (Remicade; Janssen Biologics B.V., Leiden, the Netherlands) was radiolabelled with ${ }^{99 \mathrm{~m}} \mathrm{Tc}$ in the radiopharmacy of the St Antonius Hospital using the 2-mercaptoethanol reduction method as reported elsewhere [21]. Anterior and posterior images of the thorax and abdomen were acquired $6 \mathrm{~h}$ and $20 \mathrm{~h}$ after intravenous administration of $370 \mathrm{MBq}$ of ${ }^{99 \mathrm{~m}} \mathrm{Tc}$-infliximab $(\sim 150 \mu \mathrm{g}$ antibody) according to previous 
experience, as well as a single photon emission computed tomography (SPECT)/CT of the thorax. Planar images and SPECT/CT were obtained by using a double-headed $\gamma$-camera (Siemens Healthcare, Breda, the Netherlands) with an acquisition time of $100 \mathrm{~s}$ for the $6 \mathrm{~h}$ images and $514 \mathrm{~s}$ for the $20 \mathrm{~h}$ images using a $256 \times 256$ matrix. For SPECT, a $128 \times 128$ matrix was used with 32 angles and 60 s per angle.

\section{Image analysis}

Positive uptake of ${ }^{99} \mathrm{~m}$ Tc-infliximab in the target tissue was defined as activity in either the lung parenchyma, hila or mediastinum at $6 \mathrm{~h}$ or $20 \mathrm{~h}$ that exceeded the activity in the background. A semiquantitative analysis of the SPECT images was performed by drawing a volume of interest (VoI) in the target, based on CT, and dividing by a matching VoI in the humerus or the blood compartment (aorta descendens at the carina level), thus obtaining target/background (T/B) values. VoI measurements were performed at four standardised locations in the right lung parenchyma at the carina level, lymph nodes in the left and right hila and subcarinal lymph nodes. This analysis was based on previous experience with rheumatoid arthritis, with the addition of the aorta descendens due to the high blood perfusion of the relevant target tissues in sarcoidosis. To evaluate possible selective accumulation of ${ }^{99 \mathrm{~m}} \mathrm{Tc}$-infliximab at the target level the ratios of the T/B values at $6 \mathrm{~h}$ and $20 \mathrm{~h}$ were calculated. Additional VoI measurements in ${ }^{99} \mathrm{~m}$ Tc-infliximab SPECT were performed in the lung parenchyma corresponding with the highest activity on ${ }^{18}$ F-FDG PET/CT, as well as extrathoracic activity on ${ }^{18}$ F-FDG PET/CT.

\section{Statistical analysis}

Statistical analysis was performed using statistics software (Statistical Package for the Social Sciences 22.0; IBM, Armonk, NY, USA).

The ratio of T/B values was compared to 1.00 using a one-sample t-test. Spearman's test was used for correlation analysis. Correlations were calculated between the ratio of the T/B values at the right lung parenchyma at the carina level, lymph nodes in the left and right hila and the average of both and subcarinal lymph nodes and the following parameters: serum ACE, ACE Z-score, sIL-2R, BAL CD4/CD8 ratio, VC, forced expiratory volume in $1 \mathrm{~s}$ (FEV1), Tiffeneau index (FEV1/FVC $\times 100 \%)$ and DLCO. An additional Bonferroni correction of the statistical significance level was performed, based on a total of 13 hypotheses.

In a separate analysis correlations were calculated between the ratio of $\mathrm{T} / \mathrm{B}$ values and ${ }^{18} \mathrm{~F}$-FDG maximum standardised uptake (SUVmax) values at the lymph nodes in the left and right hila and the subcarinal lymph nodes. Furthermore, correlations were calculated between the ratio of $\mathrm{T} / \mathrm{B}$ values and ${ }^{18} \mathrm{~F}$-FDG SUVmax values in the lung parenchyma zones with highest activity on ${ }^{18} \mathrm{~F}$-FDG PET/CT. Reference correlations were calculated between serum TNF- $\alpha$ values and all laboratory and clinical parameters as well as between ${ }^{18}$ F-FDG SUVmax values and all laboratory and clinical parameters.

\section{Results}

The patient characteristics at baseline are summarised in table 1 .

Images at both $6 \mathrm{~h}$ and $20 \mathrm{~h}$ were characterised by high vascular activity. Correlation with the humerus background showed positive uptake at all four target locations at both $6 \mathrm{~h}$ and $20 \mathrm{~h}$ with the highest T/B values in the left and right hilar lymph nodes (5.3 and 5.1, respectively, at $6 \mathrm{~h}$ and 4.7 and 4.5 , respectively, at $20 \mathrm{~h}$ ). The $\mathrm{T} / \mathrm{B}$ values with aorta descendens background were $<1$ for all four target locations. Figure 1 shows the CT, SPECT and fusion images of patients 1 and 2.

The T/B values (background: aorta descendens) increased between $6 \mathrm{~h}$ and $20 \mathrm{~h}$ at all four locations. A more pronounced increase (ratio of T/B values at $6 \mathrm{~h}$ and $20 \mathrm{~h}$ ) was observed in patients with an indication for systemic treatment: $+51 \%$ (left hilar lymph node, $\mathrm{p}=0.038$ ); $+46 \%$ (right hilar lymph node, $\mathrm{p}=0.025$ ); $+43 \%$ (subcarinal lymph node, $\mathrm{p}=0.056$ ); and $+26 \%$ (right lung parenchyma at the carina level, $\mathrm{p}=0.030$ ). In patients without a need for systemic treatment, no statistical significance was reached: $+30 \%$ (left hilar lymph node, $\mathrm{p}=0.12$ ); $+40 \%$ (right hilar lymph node, $\mathrm{p}=0.15$ ); $+19 \%$ (subcarinal lymph node, $\mathrm{p}=0.36$ ); and $+9 \%$ (right lung parenchyma at the carina level, $\mathrm{p}=0.70)$. Overall, the greatest increase was observed at the left and right hilar lymph nodes (mean increase $+40 \%(p=0.006)$ and $+43 \%(p=0.007)$, respectively). The ratio of T/B values differed between subjects. For the left and right hilar lymph nodes the ratios were in the range $1.04-2.01$ and $1.03-2.27$, respectively.

Positive correlations were found between the ratio of T/B values (background: aorta descendens) and all four laboratory parameters (sIL-2R concentration, serum ACE, ACE Z-score and the BAL CD4/CD8 ratio). The correlation analysis between the sIL-2R concentration and the ratio of T/B values at the four locations is depicted in figure 2 and a complete summary of the correlation analysis is provided in table 2. Correlation analysis reached statistical significance in three out of four (left hilar lymph node), four out of 
TABLE 1 Patient characteristics at baseline

\begin{tabular}{|c|c|c|c|c|c|c|c|c|c|c|c|c|c|}
\hline Patient & Sex & $\begin{array}{l}\text { Age } \\
\text { years }\end{array}$ & $\begin{array}{l}\text { Scadding } \\
\text { stage }\end{array}$ & $\begin{array}{c}\text { ACE } \\
U \cdot L^{-1}\end{array}$ & $\begin{array}{c}\text { ACE } \\
\text { Z-score }\end{array}$ & $\begin{array}{c}\mathrm{sIL}-2 \mathrm{R} \\
\mathrm{pg} \cdot \mathrm{mL}^{-1}\end{array}$ & $\begin{array}{c}\text { Serum } \\
\text { TNF-a } \\
\mathrm{pg} \cdot \mathrm{mL}^{-1}\end{array}$ & $\begin{array}{l}\text { BAL } \\
\text { CD4/ } \\
\text { CD8 } \\
\text { ratio }\end{array}$ & $\begin{array}{l}\text { VC \% } \\
\text { pred }\end{array}$ & $\begin{array}{l}\text { FEV1 } \\
\% \\
\text { pred }\end{array}$ & $\begin{array}{c}\text { Tiffeneau } \\
\text { index }\end{array}$ & $\begin{array}{c}\text { DLco } \\
\% \\
\text { pred }\end{array}$ & $\begin{array}{l}\text { Indication } \\
\text { for systemic } \\
\text { treatment }\end{array}$ \\
\hline 1 & $\mathrm{~F}$ & 53 & 1 & 48 & 1.42 & 3164 & 19.2 & 2.4 & 99.5 & 106.9 & 90.0 & 117.8 & No \\
\hline 2 & $M$ & 52 & 2 & 159 & 8.36 & 25000 & 111 & 5.8 & 82.9 & 79.3 & 76.4 & 65.3 & Yes \\
\hline 3 & $M$ & 41 & 1 & 72 & 1.11 & 6888 & 17.4 & 2.4 & 85.2 & 88.1 & 81.7 & 81.0 & Yes \\
\hline 6 & $\mathrm{~F}$ & 38 & 4 & 40 & 1.10 & 6443 & 13.3 & 5.3 & 49.2 & 44.6 & 77.8 & 45.6 & Yes \\
\hline 7 & $M$ & 57 & 0 & 101 & 3.24 & 5275 & 24.3 & 3.5 & 115.0 & 113.3 & 76.5 & 102.9 & No \\
\hline 8 & $\mathrm{~F}$ & 65 & 2 & $<5^{\S}$ & $\S$ & 20700 & 31.5 & 9.7 & 97.2 & 75.0 & 65.7 & 69.5 & No \\
\hline 9 & $M$ & 57 & 1 & 74 & f & 8247 & 46.1 & 2.3 & 102.9 & 112.9 & 83.9 & 69.3 & No \\
\hline 10 & $\mathrm{~F}$ & 31 & 2 & 85 & 2.20 & 5284 & 13.9 & 7.6 & 111.4 & 117.3 & 90.6 & 86.9 & No \\
\hline
\end{tabular}

Data are presented as $n$, unless otherwise stated. ACE: angiotensin-converting enzyme; sIL-2R: soluble interleukin-2 receptor; TNF: tumour necrosis factor; BAL: bronchoalveolar lavage; VC: vital capacity; FEV1: forced expiratory volume in $1 \mathrm{~s} ;$ DLco: diffusing capacity of the lung for carbon monoxide; F: female; M: male. ${ }^{\#}$ : ACE insertion-/deletion-corrected ACE activity; ": FEV1/forced vital capacity $\times 100 ;{ }^{+}$: no BAL performed; ${ }^{\S}$ : patient using an ACE inhibitor; ${ }^{f}$ : no Z-score determined.

four (right hilar lymph node and average of both hilar lymph nodes), one out of four (subcarinal lymph node) and none out of four (lung parenchyma) analyses. After Bonferroni correction only the correlation between the sIL-2R concentration and left hilar lymph node, right hilar lymph node and the average of both hilar lymph nodes reached statistical significance.

Negative correlations were found between the ratio of T/B values (background: aorta descendens) and all four clinical (lung function) parameters (VC, FEV1, Tiffeneau index and DLCO). The correlation analysis

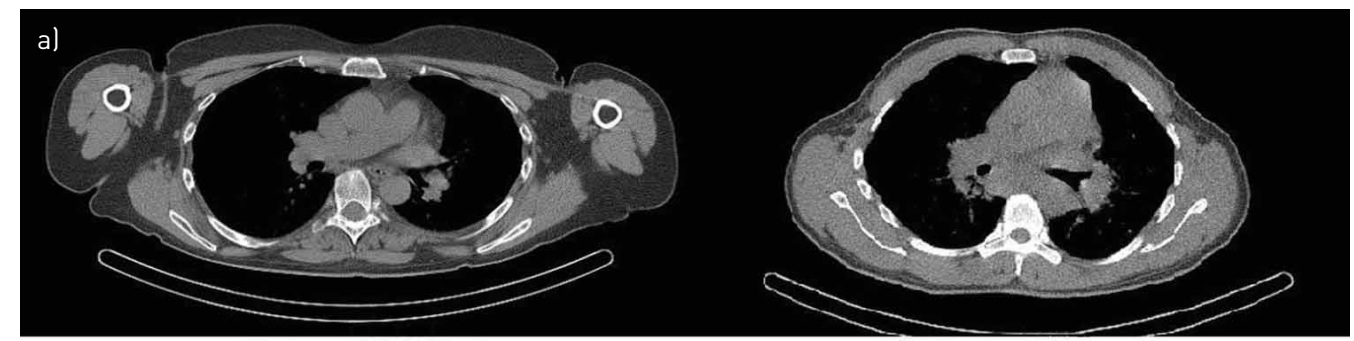

b)
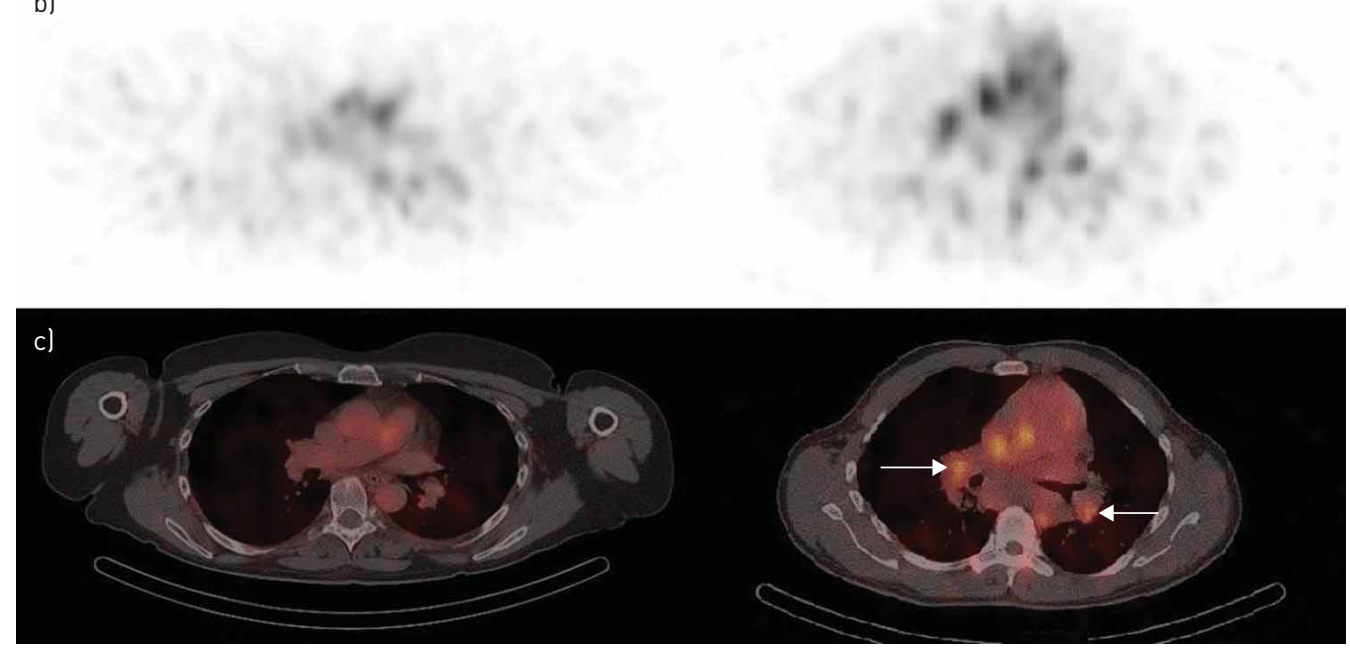

FIGURE 1 a) Computed tomography (CT), b) single photon emission computed tomography (SPECT) and c) SPECT/CT images of patient 1 (left) and patient 2 (right). Arrows indicate focal activity in the hilar lymph nodes, which is absent in patient 1. 

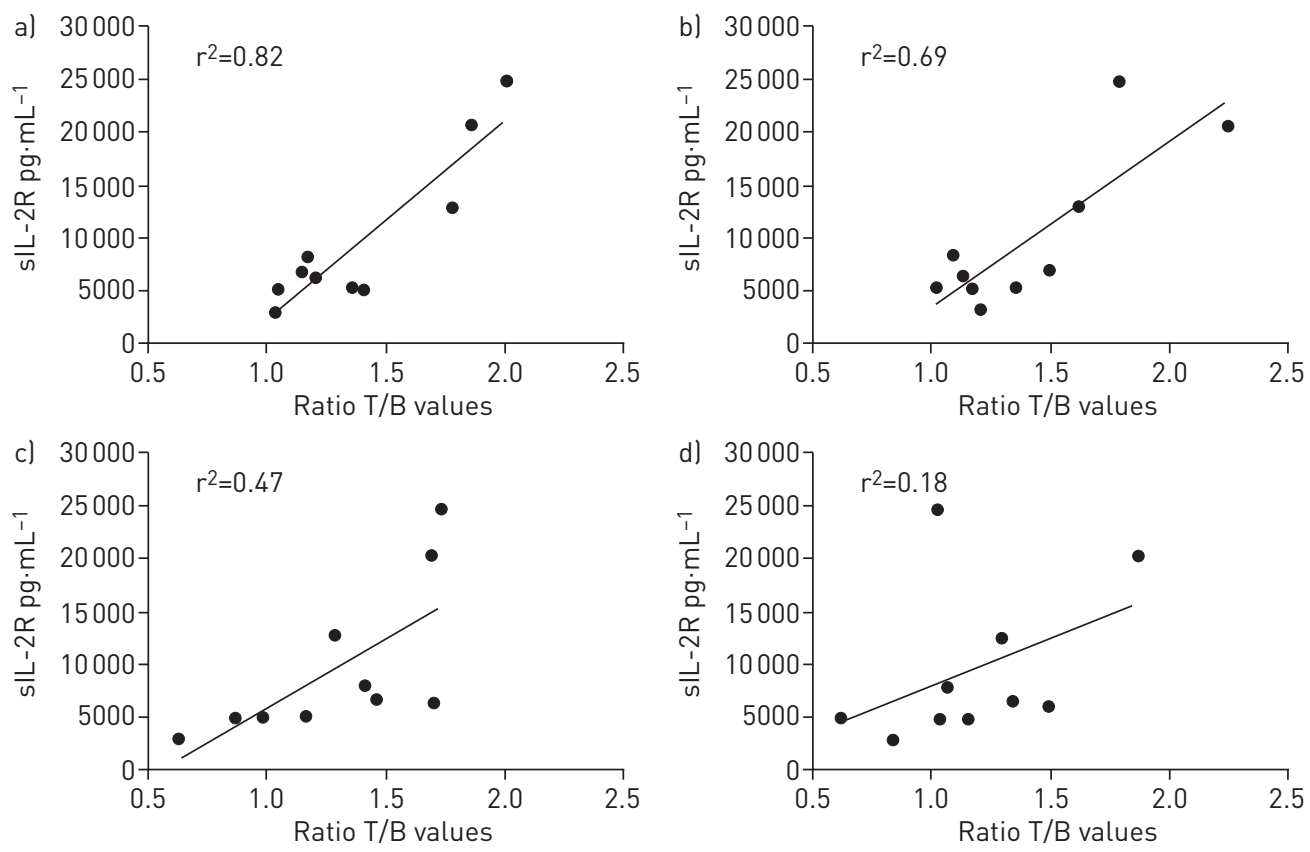

FIGURE 2 Correlation analysis of the soluble interleukin-2 receptor (sIL-2R) concentration and the ratio of target/background (T/B) values (background: aorta descendens) at a) the left hilar lymph node; b) the right hilar lymph node; c) the subcarinal lymph node; and d) the lung parenchyma at the carina level.

between $\mathrm{FEV}_{1}$ and the ratio of T/B values at the four locations is depicted in figure 3 and a complete summary of the correlation analysis is provided in table 2. Correlation analysis reached statistical significance in one out of four analyses (left hilar lymph node, average of both lymph nodes, subcarinal lymph node and the lung parenchyma) and none out of four (right hilar lymph node).

Positive correlation was found between the serum TNF- $\alpha$ level and the sIL-2R concentration, the serum ACE concentration and with the ACE Z-score. No correlations were found between the serum TNF- $\alpha$ level and the BAL CD4/CD8 ratio or any of the clinical parameters.

Based on the observed correlations between the ratio of T/B values and sIL-2R, serum ACE and ACE Z-score, further correlation analysis was performed between sIL-2R, serum ACE, ACE Z-score and the

TABLE 2 Summary of correlation analysis with anti-tumour necrosis factor (TNF)- $\alpha$ scintigraphy

\begin{tabular}{|c|c|c|c|c|c|c|c|c|c|c|c|c|}
\hline & \multicolumn{10}{|c|}{ Ratio T/B values ( $20 \mathrm{~h} / 6 \mathrm{~h}$, versus aorta descendens) anti-TNF- $\alpha$ scintigraphy } & \multicolumn{2}{|c|}{ Serum TNF- $\alpha$} \\
\hline Serum ACE & 0.76 & 0.017 & 0.73 & 0.025 & 0.78 & 0.012 & 0.41 & 0.27 & 0.20 & 0.61 & 0.80 & 0.011 \\
\hline ACE Z-score $\#$ & 0.81 & 0.015 & 0.74 & 0.038 & 0.81 & 0.014 & 0.38 & 0.35 & 0.011 & 0.98 & 0.70 & 0.052 \\
\hline Vc & -0.29 & 0.42 & -0.12 & 0.74 & -0.21 & 0.56 & -0.43 & 0.21 & -0.49 & 0.15 & -0.072 & 0.84 \\
\hline FEV 1 & -0.48 & 0.16 & -0.39 & 0.27 & -0.45 & 0.19 & -0.57 & 0.088 & -0.64 & 0.047 & -0.12 & 0.74 \\
\hline Tiffeneau index ๆ & -0.64 & 0.046 & -0.63 & 0.051 & -0.66 & 0.037 & -0.62 & 0.058 & -0.54 & 0.10 & -0.28 & 0.44 \\
\hline DLco & -0.49 & 0.15 & -0.38 & 0.28 & -0.45 & 0.19 & -0.77 & 0.009 & -0.56 & 0.093 & -0.32 & 0.38 \\
\hline
\end{tabular}



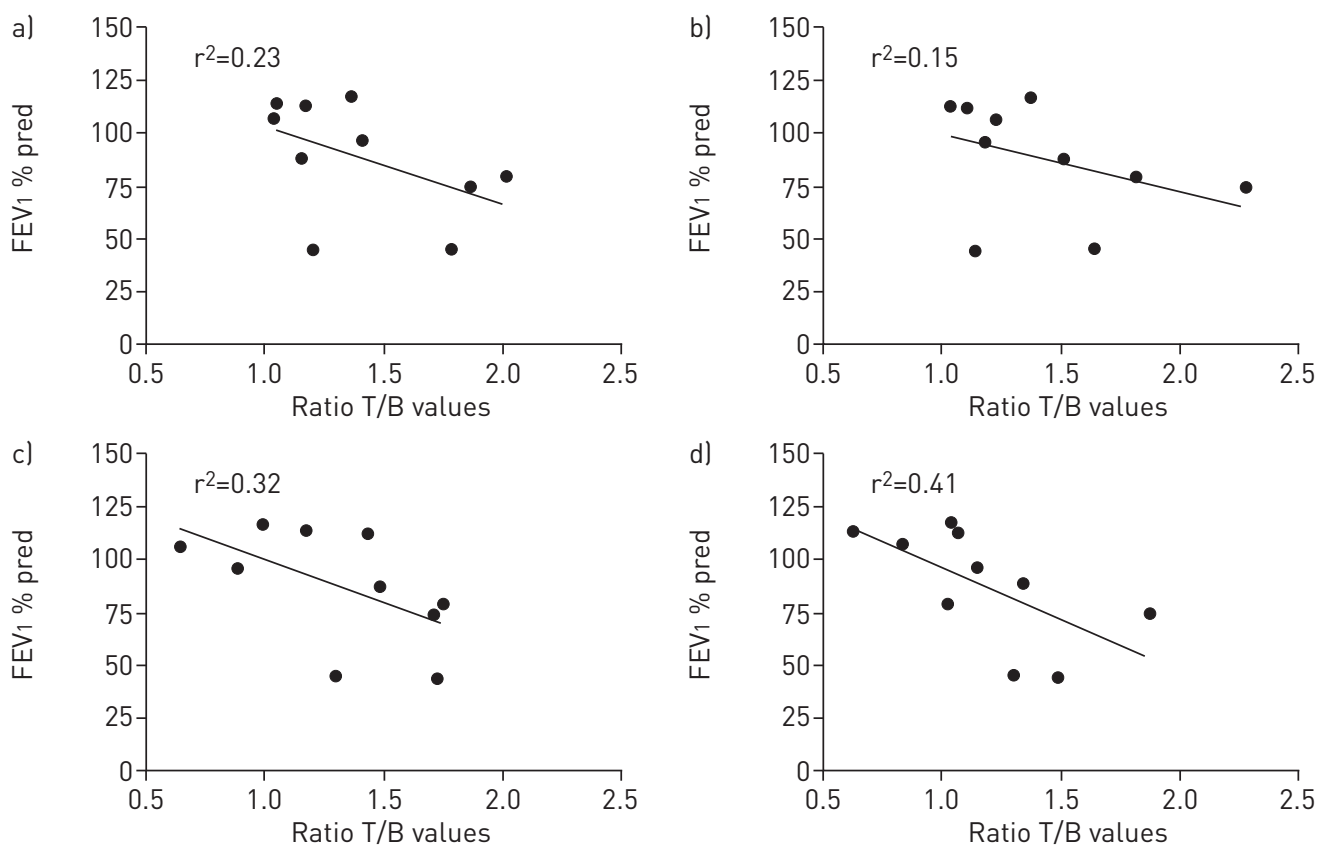

FIGURE 3 Correlation analysis of forced expiratory volume in $1 \mathrm{~s}\left(\mathrm{FEV}_{1}\right)$ and the ratio of target/backgroud (T/B) values (background: aorta descendens) at a) the left hilar lymph node; b) the right hilar lymph node; c) the subcarinal lymph node; and d) the lung parenchyma at the carina level.

clinical parameters. Negative or no correlations were found between the VC and sIL-2R $(r=-0.19, p=0.61)$, serum ACE $(r=-0.006, p=0.99)$ and ACE $Z$-score $(r=-0.31, p=0.46)$. Negative correlations were found between the FEV1 and sIL-2R $(r=-0.40, p=0.25)$, serum ACE $(r=-0.16, p=0.69)$ and ACE Z-score $(\mathrm{r}=-0.45, \mathrm{p}=0.26)$. Negative correlations were found between the Tiffeneau index and sIL-2R ( $\mathrm{r}=-0.65$, $\mathrm{p}=0.043)$, serum ACE $(\mathrm{r}=-0.58, \mathrm{p}=0.11)$ and the ACE $\mathrm{Z}$-score $(\mathrm{r}=-0.76, \mathrm{p}=0.030)$. Negative correlations were found between the DLCO and sIL-2R $(r=-0.50, p=0.14)$, serum ACE $(r=-0.40, p=0.28)$ and the ACE Z-score $(\mathrm{r}=-0.61, \mathrm{p}=0.11)$.

The results of ${ }^{18}$ F-FDG PET/CT are summarised in table 3. Correlation analysis between ${ }^{18} \mathrm{~F}-\mathrm{FDG}$ PET/ CT SUVmax values and all laboratory and all clinical parameters yielded a statistical significant correlation in two out of 32 analyses. No statistically significant correlations were observed between ${ }^{18}$ F-FDG PET/CT SUVmax values and the ratio of T/B values. Extrathoracic activity on ${ }^{18} \mathrm{~F}$-FDG PET/CT was confirmed using ${ }^{99 \mathrm{~m}} \mathrm{Tc}$-infliximab scintigraphy in one patient only (patient 2 : low activity observed in the liver hilus) and therefore no correlation analysis was performed. A summary of all correlation analyses is provided in table 4.

TABLE 3 Summary of ${ }^{18} \mathrm{~F}$-fluorodeoxyglucose (FDG) positron emission tomography (PET)/ computed tomography (CT) results

\begin{tabular}{lccc} 
Patient & \multicolumn{3}{c}{ SUVmax ${ }^{\mathbf{1 8}}$ F-FDG PET/CT } \\
\cline { 2 - 4 } & Hila/mediastinum & Lung parenchyma & Extrathoracic (location) \\
\hline $\mathbf{1}$ & 17.3 & 1.1 & NA \\
$\mathbf{2}$ & 12.9 & 3.3 & 8.8 (liver); 13.1 (lymph node) \\
$\mathbf{3}$ & 23.4 & 10.5 & 4.9 (lymph node); 12.6 (interventricular septum) \\
$\mathbf{4}$ & 12.8 & 1.2 & 12.6 (lymph node) \\
$\mathbf{5}$ & 6.9 & 6.1 & 4.3 (lymph node) \\
$\mathbf{6}$ & 8.0 & 10.3 & 6.4 (lymph node) \\
$\mathbf{7}$ & 7.1 & 1.1 & NA \\
$\mathbf{8}$ & 14.7 & 6.9 (one small nodule) & 6.5 (lymph node) \\
$\mathbf{9}$ & 5.6 & 1.1 & NA \\
$\mathbf{1 0}$ & 13.3 & 3.4 (subpleural) & 5.2 (lymph node)
\end{tabular}

SUVmax: maximum standardised uptake value; NA: not applicable. 
TABLE 4 Summary of correlation analysis with ${ }^{18} \mathrm{~F}$-fluorodeoxyglucose (FDG) positron emission tomography (PET)/computed tomography (CT)

\begin{tabular}{|c|c|c|c|c|c|c|c|c|}
\hline & \multicolumn{8}{|c|}{ SUVmax ${ }^{18}$ F-FDG PET/CT } \\
\hline & $\begin{array}{l}\text { Left } \\
\text { hilar } \\
\text { lymph } \\
\text { node }\end{array}$ & p-value & $\begin{array}{l}\text { Right } \\
\text { hilar } \\
\text { lymph } \\
\text { node }\end{array}$ & p-value & $\begin{array}{l}\text { Subcarinal } \\
\text { lymph node }\end{array}$ & p-value & $\begin{array}{c}\text { Lung } \\
\text { parenchyma }\end{array}$ & p-value \\
\hline Serum ACE & -0.37 & 0.33 & -0.18 & 0.64 & -0.19 & 0.63 & -0.093 & 0.81 \\
\hline ACE Z-score ${ }^{\#}$ & -0.56 & 0.15 & -0.41 & 0.31 & -0.42 & 0.30 & -0.025 & 0.95 \\
\hline sIL-2R & -0.19 & 0.60 & 0.025 & 0.95 & -0.065 & 0.86 & 0.16 & 0.66 \\
\hline $\begin{array}{l}\text { BAL CD4/ } \\
\text { CD8 ratio }\end{array}$ & -0.088 & 0.82 & 0.041 & 0.92 & -0.070 & 0.86 & 0.32 & 0.41 \\
\hline vc & 0.18 & 0.63 & 0.19 & 0.59 & 0.18 & 0.63 & -0.65 & 0.041 \\
\hline FEV 1 & 0.22 & 0.54 & 0.19 & 0.60 & 0.24 & 0.51 & -0.70 & 0.024 \\
\hline DLco & 0.44 & 0.21 & 0.34 & 0.34 & 0.52 & 0.13 & -0.62 & 0.057 \\
\hline $\begin{array}{l}\text { Tiffeneau } \\
\text { index }\end{array}$ & 0.40 & 0.26 & 0.29 & 0.42 & 0.46 & 0.18 & -0.39 & 0.26 \\
\hline $\begin{array}{l}\text { Anti-TNF- } \alpha \\
\text { scintigraphy }\end{array}$ & -0.21 & 0.56 & 0.36 & 0.31 & -0.33 & 0.35 & -0.55 & 0.26 \\
\hline
\end{tabular}

Data are presented as $r$, unless otherwise stated. Bold type indicates $p<0.05$. SUVmax: maximum standardised uptake value; ACE: angiotensin-converting enzyme; sIL-2R: soluble interleukin-2 receptor; BAL: bronchoalveolar lavage; VC: vital capacity; FEV1: forced expiratory volume in $1 \mathrm{~s}$; DLCo: diffusing capacity of the lung for carbon monoxide; TNF: tumour necrosis factor. ${ }^{\#}$ : ACE insertion-/deletion-corrected ACE activity; ${ }^{\text {?: }} \mathrm{FEV} 1 /$ forced vital capacity $\times 100$.

\section{Discussion}

In this study we observed selective accumulation of ${ }^{99} \mathrm{~m}$ Tc-infliximab in the target tissue of pulmonary sarcoidosis patients. The accumulation was highest and most robust in the hilar lymph nodes. While some patients showed significant accumulation, others did not, which suggests varying levels of TNF- $\alpha$ expression between subjects. The most pronounced accumulation was observed in patients with an indication for systemic treatment. The accumulation of ${ }^{99 \mathrm{~m}} \mathrm{Tc}$-infliximab as measured by the ratio of $\mathrm{T} / \mathrm{B}$ values (increase between $6 \mathrm{~h}$ and $20 \mathrm{~h}$ ) correlated positively with all four laboratory parameters (sIL-2R concentration, serum ACE, ACE Z-score and the BAL CD4/CD8 ratio), while accumulation correlated negatively with all four clinical parameters (VC, FEV1, Tiffeneau index and DLCO).

The finding that TNF- $\alpha$ expression differs between sarcoidosis patients is in agreement with previous research. Asano et al. [7] measured TNF- $\alpha$ levels in lymph node samples of sarcoidosis patients and observed highest levels in lymph nodes in the active stage as opposed to the early and healing stages. FEHRENBACH et al. [5] showed that the level of bioactive TNF- $\alpha$ spontaneously released by isolated BAL immune cells differed between patients. Highest levels were observed in the presence of aggregates of alveolar macrophages, which represent predecessors to granulomas. Well-developed granulomas were associated with lower levels of TNF- $\alpha$ release, confirming that TNF- $\alpha$ is important during the initial phase of granuloma formation [5].

A variable TNF- $\alpha$ expression as measured by ${ }^{99 \mathrm{~m}} \mathrm{Tc}$-infliximab scintigraphy was also observed by GALLI et al. [22] in a similar group of newly diagnosed pulmonary sarcoidosis patients. They observed that the pulmonary accumulation of ${ }^{99 \mathrm{~m}} \mathrm{Tc}$-infliximab was very low and variable and showed no correlation with the pulmonary ${ }^{18}$ F-FDG PET/CT SUVmax values. A moderate correlation was observed with the CD4/CD8 ratio in peripheral blood lymphocytes, but not with the CD4/CD8 ratio or cellularity of BAL, possibly because of the small sample size. The authors also explained the differences between ${ }^{18} \mathrm{~F}-\mathrm{FDG}$ and ${ }^{99 m}$ Tc-infliximab by the different mechanism of uptake of the two radiopharmaceuticals and by the individual variability in the production of TNF- $\alpha$.

In the present study we expanded the scope with several clinical and laboratory parameters. Results confirmed a limited correlation between ${ }^{99 \mathrm{~m}} \mathrm{Tc}$-infliximab accumulation in the lung parenchyma and all laboratory and clinical parameters, with the highest radiopharmaceutical accumulation observed in the lymph nodes. An explanation could be a lower TNF- $\alpha$ concentration in the lung tissue, potentially caused by a washout of soluble TNF- $\alpha$ due to high perfusion. An additional explanation for the lower overall uptake in the lung parenchyma could be the normal or only mildly altered lung function observed in our population. This is confirmed by ${ }^{18}$ F-FDG PET/CT, demonstrating limited or no metabolic activity in the 
lung parenchyma in six out of 10 patients. The mild pulmonary involvement suggests a limited parenchymal TNF- $\alpha$ expression and subsequent low ${ }^{99 \mathrm{~m}} \mathrm{Tc}$-infliximab accumulation.

We observed no correlation between the accumulation of ${ }^{99 \mathrm{~m}} \mathrm{Tc}$-infliximab and ${ }^{18} \mathrm{~F}$-FDG PET/CT SUVmax values.

Our findings of ${ }^{99} \mathrm{~m}$ Tc-infliximab accumulation correlating with clinical parameters are in line with literature on rheumatoid arthritis. In a study using ${ }^{99 \mathrm{~m}} \mathrm{Tc}$-adalimumab, another TNF- $\alpha$ inhibitor, BARRERA et al. [24] found that joint uptake of ${ }^{99 \mathrm{~m}} \mathrm{Tc}$-adalimumab in five rheumatoid arthritis patients decreased after treatment with systemic corticosteroids and was indicative of a decrease in the rheumatoid arthritis disease activity score. In a similar study, RoIMICHER et al. [25] found good correlation between ${ }^{99 m}$ Tc-adalimumab uptake and magnetic resonance imaging of hands and wrists in eight rheumatoid arthritis patients. ${ }^{99 \mathrm{~m}} \mathrm{Tc}$-adalimumab scintigraphy showed a higher sensitivity than clinical examination. The value of ${ }^{99 \mathrm{~m}} \mathrm{Tc}$-infliximab scintigraphy in the prediction of the response of intra-articular infliximab in 12 rheumatoid arthritis and spondylarthropathy patients was illustrated by ConTi et al. [20]. Affected joints showed higher tracer uptake than nonaffected joints and the degree of tracer accumulation prior to therapy allowed to distinguish responders, partial responders and nonresponders. In contrast, D'AlESSANDRIA et al. [21] found that ${ }^{99 \mathrm{~m}}$ Tc-infliximab scintigraphy could not predict clinical outcome in 10 patients with Crohn's disease. Possible explanations discussed by the authors were either low TNF- $\alpha$ production in the gut of Crohn's disease patients, low ${ }^{99 \mathrm{~m}} \mathrm{Tc}$-infliximab extravasation at the radiopharmaceutical dose of $200 \mu \mathrm{g}$ or the pre-treatment with oral prednisone $\left(1 \mathrm{mg} \cdot \mathrm{kg}^{-1}\right)$ which could have resulted in downregulation of bowel TNF- $\alpha$ expression.

Serum TNF- $\alpha$ levels in serum in our patients were in the range $13.9-111 \mathrm{pg} \cdot \mathrm{mL}^{-1}$, which is in agreement with reported values [26]. Although a statistically significant correlation was found with the sIL-2R and serum ACE concentrations, no significant correlation was found with the ACE Z-score, BAL CD4/CD8 ratio or any of the clinical parameters. This finding illustrates that the measurement of circulating (soluble) TNF- $\alpha$ is not equivalent to measurement of local TNF- $\alpha$ expression in tissue that includes transmembrane TNF- $\alpha$, which plays an important role in granulomatous diseases such as sarcoidosis [27].

A correlation between accumulation of ${ }^{99} \mathrm{~m}$ Tc-infliximab and serum sIL-2R and ACE concentrations is plausible from the perspective of existing knowledge. КeICHO et al. [28] and INA et al. [29] observed higher levels of sIL-2R in serum of patients with active sarcoidosis compared with inactive disease and found a significant correlation with serum ACE levels. Alveolar macrophages are a source of sIL-2R, ACE and TNF- $\alpha[4,5,28,29]$. It therefore seems that the initial phase of granuloma formation is associated with higher levels of sIL-2R, ACE and TNF- $\alpha$, and based on the latter, a higher accumulation of ${ }^{99} \mathrm{~m}$ Tc-infliximab. Despite these associations, sIL-2R, ACE and TNF- $\alpha$ are not interchangeable. While alveolar macrophages are the main source of TNF- $\alpha$ in sarcoidosis, T-lymphocytes and monocytes also release sIL-2R, and epitheloid cells and monocytes contribute to the production of ACE $[5,29]$. In our study group, the accumulation of ${ }^{99 \mathrm{~m}} \mathrm{Tc}$-infliximab showed better correlation with clinical parameters than sIL-2R, serum ACE/ACE Z-score and serum TNF- $\alpha$.

Correlation analysis with ${ }^{18} \mathrm{~F}$-FDG PET/CT SUVmax values in our population showed limited correlation with laboratory parameters, clinical parameters or ${ }^{99 \mathrm{~m}} \mathrm{Tc}$-infliximab accumulation. These findings support that ${ }^{18}$ F-FDG PET/CT and ${ }^{99 m}$ Tc-infliximab scintigraphy are not equivalent and confirms the notion that high activity on ${ }^{18} \mathrm{~F}$-FDG PET/CT is not necessarily indicative of high TNF- $\alpha$ expression.

A limitation of our study is the small group size (10 patients) and subsequent limited statistical power. Since it involved a pilot study we chose newly diagnosed patients without prior immunosuppressive therapy to avoid the risk of suppression of TNF- $\alpha$ expression. Future research should focus on a population with an indication for anti-TNF- $\alpha$ therapy to evaluate the correlation between ${ }^{99 \mathrm{~m}} \mathrm{Tc}$-infliximab pre-therapy accumulation and treatment response.

Compared with the SUVmax values obtained with ${ }^{18} \mathrm{~F}-\mathrm{FDG}$ PET/CT the ratios of T/B values in the SPECT/ CT images obtained with ${ }^{99 \mathrm{~m}} \mathrm{Tc}$-infliximab are less pronounced. A major contribution in this respect is the high activity in the blood pool, which is due to the long biological half-life of infliximab (9 days). The short physical half-life of ${ }^{99} \mathrm{~m}$ Tc $(6 \mathrm{~h})$ necessitates scanning within $24 \mathrm{~h}$. An interesting possible way of addressing this issue is the use of a radioactive isotope with a longer physical half-life, e.g. zirconium-89 (physical half-life $78 \mathrm{~h}$ ), which has been used successfully in the radiolabelling and clinical application of other monoclonal antibodies [30]. Finally, a tool to quantify the total activity in one organ (e.g. the lungs), as opposed to the focal activity measurements used in our study, might have great clinical value [31]. If such tools become available and are properly validated, they should be used in future studies.

Our population consisted of pulmonary sarcoidosis patients. WIJNEN et al. [19] recently discussed that the higher infliximab response rate observed for sarcoidosis patients suffering from uveitis is probably 
determined by the shorter time between diagnosis and initiation of anti-TNF- $\alpha$ treatment compared with small-fibre neuropathy and respiratory functional impairment. At least a partial explanation may be that TNF- $\alpha$ expression is higher during early granuloma formation phases. As our results illustrate that serum TNF- $\alpha$ levels correlate poorly with clinical parameters, it will be important to quantify TNF- $\alpha$ expression in tissue, including transmembrane TNF- $\alpha$, e.g. by means of scintigraphic measurement. Early intervention with infliximab as part of an aggressive treatment has already been investigated for early rheumatoid arthritis and has been proven to be beneficial, although the increased effects were sometimes limited [32, 33]. An aggressive anti-TNF- $\alpha$ intervention strategy for clinically threatened sarcoidosis patients with the highest TNF- $\alpha$ expression, even as second- or first-line therapy, has the potential of increased efficacy, prevention of disease progression and higher cost-effectiveness.

In conclusion, our results show that a variable, selective accumulation of ${ }^{99 \mathrm{~m}} \mathrm{Tc}$-infliximab occurs in newly diagnosed sarcoidosis patients, mainly in the hilar lymph nodes. Thus, ${ }^{99 \mathrm{~m}} \mathrm{Tc}$-infliximab accumulation reflects the TNF- $\alpha$ expression in each individual patient and therefore provides valuable information on the presence of the biological target for anti-TNF- $\alpha$ therapy. Further research is warranted to evaluate the role of ${ }^{99 \mathrm{~m}} \mathrm{Tc}$-infliximab scintigraphy in predicting anti-TNF- $\alpha$ therapy response in sarcoidosis patients.

\section{Acknowledgements}

The authors wish to thank Mirjam Hoppers (St Antonius Hospital, Nieuwegein, the Netherlands) for her assistance with the screening of patients and Imarda de Bruin and Kees de Bruijn (St Antonius Hospital) for their assistance with the preparation and quality control of ${ }^{99 \mathrm{~m}} \mathrm{Tc}$-infliximab.

\section{References}

1 Statement on sarcoidosis. Joint Statement of the American Thoracic Society (ATS), the European Respiratory Society (ERS) and the World Association of Sarcoidosis and Other Granulomatous Disorders (WASOG) adopted by the ATS Board of Directors and by the ERS Executive Committee, February 1999. Am J Respir Crit Care Med 1999; 160: 736-755

2 Grutters JC, van den Bosch JM. Corticosteroid treatment in sarcoidosis. Eur Respir J 2006; 28: 627-636.

3 Paramothayan NS, Lasserson TJ, Walters EH. Immunosuppressive and cytotoxic therapy for pulmonary sarcoidosis. Cochrane Database Syst Rev 2006; 3: CD003536.

4 Iannuzzi MC, Fontana JR. Sarcoidosis: clinical presentation, immunopathogenesis, and therapeutics. JAMA 2011; 305: 391-399.

5 Fehrenbach H, Zissel G, Goldmann T, et al. Alveolar macrophages are the main source for tumour necrosis factor- $\alpha$ in patients with sarcoidosis. Eur Respir J 2003; 21: 421-428.

6 Ruco LP, Stoppacciaro A, Pomponi D, et al. Immunoreactivity for IL-1 beta and TNF alpha in human lymphoid and nonlymphoid tissues. Am J Pathol 1989; 135: 889-897.

7 Asano M, Minagawa T, Ohmichi M, et al. Detection of endogenous cytokines in sera or in lymph nodes obtained from patients with sarcoidosis. Clin Exp Immunol 1991; 84: 92-96.

8 Braun J, Baraliakos X, Listing J, et al. Persistent clinical efficacy and safety of anti-tumour necrosis factor $\alpha$ therapy with infliximab in patients with ankylosing spondylitis over 5 years: evidence for different types of response. Ann Rheum Dis 2008; 67: 340-345.

9 Maini R, St Clair EW, Breedveld F, et al. Infliximab (chimeric anti-tumour necrosis factor $\alpha$ monoclonal antibody) versus placebo in rheumatoid arthritis patients receiving concomitant methotrexate: a randomised phase III trial. ATTRACT Study Group. Lancet 1999; 354: 1932-1939.

10 Rutgeerts P, Sandborn WJ, Feagan BG, et al. Infliximab for induction and maintenance therapy for ulcerative colitis. N Engl J Med 2005; 353: 2462-2476.

11 Baughman RP, Drent M, Kavuru M, et al. Infliximab therapy in patients with chronic sarcoidosis and pulmonary involvement. Am J Respir Crit Care Med 2006; 174: 795-802.

12 Rossman MD, Newman LS, Baughman RP, et al. A double-blinded, randomized, placebo-controlled trial of infliximab in subjects with active pulmonary sarcoidosis. Sarcoidosis Vasc Diffuse Lung Dis 2006; 23: 201-208.

13 Judson MA, Baughman RP, Costabel U, et al. Efficacy of infliximab in extrapulmonary sarcoidosis: results from a randomised trial. Eur Respir J 2008; 31: 1189-1196.

14 van Rijswijk HNAJ, Vorselaars ADM, Ruven HJT, et al. Changes in disease activity, lung function and quality of life in patients with refractory sarcoidosis after anti-TNF treatment. Expert Opin Orphan Drugs 2013; 1: 437-443.

15 Hostettler KE, Studler U, Tamm M, et al. Long-term treatment with infliximab in patients with sarcoidosis. Respiration 2012; 83: 218-224.

16 Russell E, Luk F, Manocha S, et al. Long term follow-up of infliximab efficacy in pulmonary and extra-pulmonary sarcoidosis refractory to conventional therapy. Semin Arthritis Rheum 2013; 43: 119-124.

17 Sweiss NJ, Barnathan ES, Lo K, et al. C-reactive protein predicts response to infliximab in patients with chronic sarcoidosis. Sarcoidosis Vasc Diffuse Lung Dis 2010; 27: 49-56.

18 Loza MJ, Brodmerkel C, Du Bois RM, et al. Inflammatory profile and response to anti-tumor necrosis factor therapy in patients with chronic pulmonary sarcoidosis. Clin Vaccine Immunol 2011; 18: 931-939.

19 Wijnen PA, Cremers JP, Nelemans PJ, et al. Association of the TNF- $\alpha$ G-308A polymorphism with TNF-inhibitor response in sarcoidosis. Eur Respir J 2014; 43: 1730-1739.

20 Conti F, Malviya G, Ceccarelli F, et al. Role of scintigraphy with ${ }^{99 \mathrm{~m}} \mathrm{Tc}$-infliximab in predicting the response of intraarticular infliximab treatment in patients with refractory monoarthritis. Eur J Nucl Med Mol Imaging 2012; 39: 1339-1347.

21 D'Alessandria C, Malviya G, Viscido A, et al. Use of a ${ }^{99 m}$ Tc labeled anti-TNF $\alpha$ monoclonal antibody in Crohn's disease: in vitro and in vivo studies. Q J Nucl Med Mol Imaging 2007; 51: 334-342. 
22 Galli F, Lanzolla $\mathrm{T}$, Pietrangeli V, et al. In vivo evaluation of TNF-alpha in the lungs of patients affected by sarcoidosis. Biomed Res Int 2015; 2015: 401341

23 Kruit A, Grutters JC, Gerritsen WBM, et al. ACE I/D-corrected Z-scores to identify normal and elevated ACE activity in sarcoidosis. Respir Med 2007; 101: 510-515.

24 Barrera P, Oyen WJG, Boerman OC, et al. Scintigraphic detection of tumour necrosis factor in patients with rheumatoid arthritis. Ann Rheum Dis 2003; 62: 825-828.

25 Roimicher L, Lopes FP, de Souza SA, et al. ${ }^{99 \mathrm{~m}} \mathrm{Tc}$-anti-TNF- $\alpha$ scintigraphy in RA: a comparison pilot study with MRI and clinical examination. Rheumatology 2011; 50: 2044-2050.

26 Beirne P, Pantelidis P, Charles P, et al. Multiplex immune serum biomarker profiling in sarcoidosis and systemic sclerosis. Eur Respir J 2009; 34: 1376-1382.

27 Horiuchi T, Mitoma H, Harashima S, et al. Transmembrane TNF- $\alpha$ : structure, function and interaction with anti-TNF agents. Rheumatology 2010; 49: 1215-1228.

28 Keicho N, Kitamura K, Takaku F, et al. Serum concentration of soluble interleukin-2 receptor as a sensitive parameter of disease activity in sarcoidosis. Chest 1990; 98: 1125-1129.

29 Ina Y, Takada K, Sato T, et al. Soluble interleukin 2 receptors in patients with sarcoidosis. Possible origin. Chest 1992; 102: 1128-1133.

30 Dijkers EC, Oude Munnink TH, Kosterink JG, et al. Biodistribution of ${ }^{89} \mathrm{Zr}$-trastuzumab and PET imaging of HER2-positive lesions in patients with metastatic breast cancer. Clin Pharmacol Ther 2010; 87: 586-592.

31 Keijsers RGM, van den Heuvel DAF, Grutters JC. Imaging the inflammatory activity of sarcoidosis. Eur Respir J 2013; 41: 743-751.

32 Breedveld FC, Emery P, Keystone E, et al. Infliximab in active early rheumatoid arthritis. Ann Rheum Dis 2004; 63: $149-155$.

33 Leirisalo-Repo M, Kautiainen H, Laasonen L, et al. Infliximab for 6 months added on combination therapy in early rheumatoid arthritis: 2-year results from an investigator-initiated, randomised, double-blind, placebo-controlled study (the NEO-RACo Study). Ann Rheum Dis 2013; 72: 851-857. 